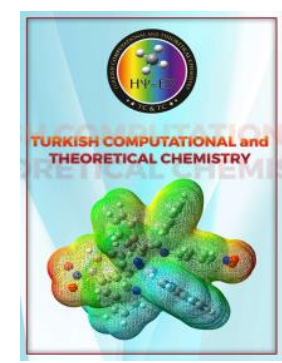

Received: 08.08.2020
Turkish Computational and Theoretical Chemistry

Turkish Comp Theo Chem (TC\&TC)

Volume(Issue): 4(2) - Year: 2020 - Pages: 67-75

e-ISSN: 2602-3237

https://doi.org/10.33435/tcandtc. 768758

Accepted: 14.09.2020

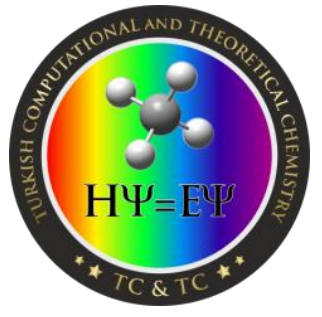

Research Article

\title{
Computational determination the reactivity of salbutamol and propranolol drugs
}

\author{
Rebaz A. Omar ${ }^{l, a, d}$, Pelin Koparir ${ }^{\text {b }}$, Lana O. Ahmed ${ }^{\text {cee }}$, Matin Koparir ${ }^{\text {d }}$ \\ ${ }^{a}$ Department of Chemistry, Faculty of Science \& Health, Koya University, Koya KOY45. KRG \\ ${ }^{\mathrm{b}}$ Institute of Forensics, Department of Chemistry, Malatya, TURKEY \\ ${ }^{\mathrm{c}}$ Department of Physics, Faculty of Science \& Health, Koya University, Koya KOY45. KRG \\ ${ }^{d}$ Firat University, Faculty of Science, Department of Chemistry, 23169 Elazig, Turkiye. \\ ${ }^{\mathrm{e}}$ Firat University, Faculty of Science, Department of Physics, 23169 Elazig, Turkiye.
}

\begin{abstract}
Gaussian software programs 09 was utilized to find the reactivity of salbutamol (SAL) and propranolol (PRO). Density Functional Theory (DFT) and Hartree-Fock (HF) were used to determine the energy band gaps. B3LYP/6-31++G(d,p) lower energy level was chosen as the base set. Geometrical structures with frontier molecular orbitals estimation for both the SAL and PRO. Atomic charge distribution and molecular electrostatic potential evaluation were performed for both drugs. For thermodynamic analysis Ab-initio DFT with HF at 6-31++G base sets were accomplished. The results showed that the PRO is more reactive than SAL.
\end{abstract}

Keywords: Salbutamol (SAL), Propranolol (PRO), Density Functional Theory (DFT), Hartree-Fock (HF), Frontier molecular orbitals.

\section{Graphical Abstract}

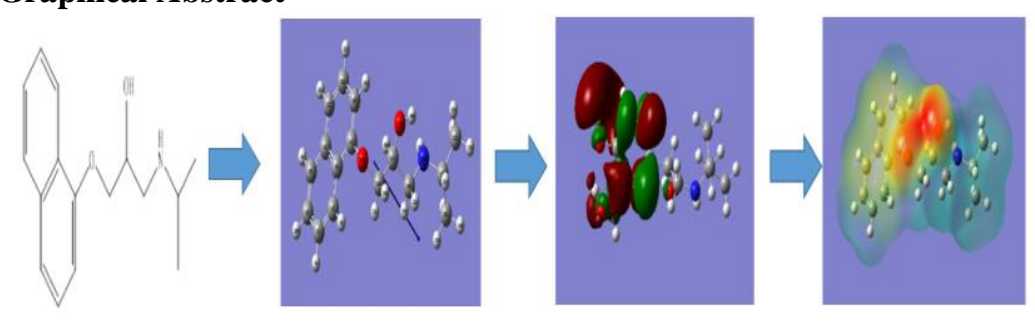

\section{Introduction}

((RS)-4-[2-(tert-butylamino)-1 hydroxyethyl]2-(hydroxymethyl)phenol (Figure 1A) is the IUPAC name of Salbutamol (SAL). It is a drug commonly used to treat asthma, chronic pulmonary disease, and potassium levels in the blood. SAL has some side effects including dizziness, headache, shakiness, and rapid heart rate [1-3], and can source serious health issues, including aggravating bronchospasm, erratic heartbeat, and low levels of potassium in the blood if given in excess or if eaten improperly [4, 5].

(1-isopropylamino-3-(naphthalen-1-yloxy) propan-2-ol is the IUPAC name for Propranolol (PRO) (Figure 1B) is a blocking agent of beta- adrenergic [6-8]. This drug is widely used for the diagnosis of high blood pressure, chronic angina pectoris, prophylaxis, and cardiac arrhythmias, myocardial reinfections prophylaxis, and tremor treatment $[7,8]$. PRO can cause negative reactions, such as heart failure, exacerbation of atrioventricular conduction disorders, bronchospasm, hypotension, and extreme bradycardia [7, 8].

(SAL and PRO) are two drugs not commonly used, since PRO is used alone not with a cardioselctive beta-blocking agent and it is not possible to be used with SAL because the risk was higher, can outweigh the benefits for asthma patients, and should be prevented or monitored by

\footnotetext{
${ }^{1}$ Corresponding Authors

e-mail: rebaz.anwar@koyauniversity.org
} 
a doctor [9-11]. In previous literature was motioned that some patients symptomatic affected by an overdose of SAL. While PRO was used as an antidote and anti-asthmatic drugs [12]. Ramoska et al. documented the used of PRO in two asthmatic patients to treat SAL toxicity, in which case PRO was used to mitigate the impact caused by SAL [13]. Kupel [14] used PRO for infant hemangiomas but only 3 out of 14 patients had bronchospasm and had treatment with SAL, so while SAL and PRO are not present in pharmaceutical formulations together, they can be founder-administered in clinical treatments $[12,13]$.

Concurrent determination of SAL and PRO is still very important for physiological pharmacology and diagnosable disorder in biomedical fluids [15]. Those drugs in a previous study have extremes cases, this is due to mismanagement or poisoning. Some procedures for the simultaneous determination of these groups of drugs have been published in the literature $[16,17]$. Between these analytical methods, the electroanalytical technique demonstrated major advantages in the study of biological fluid samples compared to other conventional methods such as chromatography and spectrophotometry [18]. Its advantages include greater flexibility, real-time analysis, low cost, and fast analysis time [19-21].

In this work, computational software is using to analyze the structure, physicochemical properties to found the reactivity of both drugs.

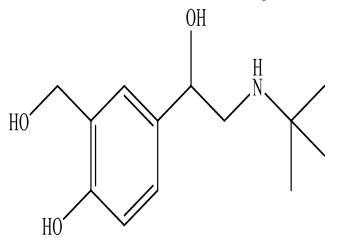

A

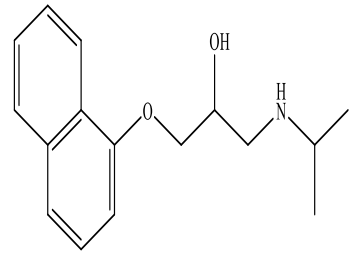

B
Figure 1. Structure of drugs (A) Salbutamol (SAL) (B) Propranolol (PRO).

\section{Computational Study}

All study is done by using Gaussian software program 09 [22], the geometric structure of (SAL) and (PRO) has been optimized by both methods Hartree-Fock (HF) and Density Functional Theory (DFT) with the different base sets [23]. Firstly, eight separately bases set were used for both (DFT $\&$ HF) to find the energy bandgaps. The secondlowest degree of energy was used for further research optimization. The geometrical structure with certain geometrical parameters was calculated for both drugs (SAL \& PRO) to confirm the analysis of the structure. Calculated Frontier Molecular Orbitals, Mulliken charge Distribution, and Molecular Electrostatic Potential using B3LYP/6-31G (d,p) base set. Thermodynamic properties for both molecules are performed.

\section{Results and Discussion}

3.1 Energy Band Gaps

The first step in this work finds the optimized molecular structure [24]. The bandgaps energy was associated with various basis sets mentioned [25] in Table 1. The energy bandgaps which were calculated for both drugs (SAL and PRO) by the difference between HOMO and LUMO energy levels, it has appeared after optimized was completely and find from MOs. Generally, the energy band gaps for the Hartree-Fock (HF) method have higher values compared with the density functional theory (DFT), as shown in Table 1. But for (PRO) drugs the energy bandgaps for both methods (HF and DFT) have a lower value than (SAL) drugs, the first result showed the PRO is more reactive than SAL. The value of the DFT methods for both drugs shows that very close to each other. 6-31++ G basis set at DFT methods was chosen for further analysis due to it has lower energy levels compared to the other basis set.

Table 1. Energy bandgaps for SAL and PRO at HF and DFT methods with different base sets.

\begin{tabular}{ccccc}
\hline \multirow{2}{*}{ Basis sets } & \multicolumn{2}{c}{ Salbutamol (SAL) } & \multicolumn{2}{c}{ Propranolol (PRO) } \\
\cline { 2 - 5 } & $\begin{array}{c}\text { HF method } \\
\text { Energy band gaps } \\
(\mathrm{eV})\end{array}$ & $\begin{array}{c}\text { DFT method } \\
\text { Energy band gaps } \\
(\mathrm{eV})\end{array}$ & $\begin{array}{c}\text { HF method } \\
\text { Energy band gaps } \\
(\mathrm{eV})\end{array}$ & $\begin{array}{c}\text { DFT method } \\
\text { Energy band gaps } \\
(\mathrm{eV})\end{array}$ \\
\hline $3-21 \mathrm{G}$ & 0.4467 & 0.1802 & 0.3889 & 0.1746 \\
$3-21+\mathrm{G}$ & 0.3811 & 0.1727 & 0.3616 & 0.1721 \\
$6-31 \mathrm{G}$ & 0.4423 & 0.1777 & 0.3830 & 0.1732 \\
$6-31+\mathrm{G}$ & 0.3756 & 0.1718 & 0.3558 & 0.1702 \\
$6-31++\mathrm{G}$ & 0.3508 & 0.1710 & 0.3223 & 0.1701 \\
$6-311 \mathrm{G}$ & 0.4365 & 0.1761 & 0.3801 & 0.1728 \\
$6-311+\mathrm{G}$ & 0.3742 & 0.1734 & 0.3487 & 0.1712 \\
$6-311++\mathrm{G}$ & 0.3510 & 0.1728 & 0.3220 & 0.1712 \\
\hline
\end{tabular}




\subsection{Geometrical Structures}

For both drugs (Figure 2) is the most stable structure optimized by B3LYP/6-31G (d,p) with determining atomic numeration and orientation in a molecule. The geometry structure of both molecules clearly shapes a distinct globular structure that exposes all reactive sites effectively to the reactive of the molecules. SAL and PRO structure conformation let a molecule get closer to the reactive molecules. Table 2 gives certain geometrical parameters for SAL and PRO drugs. In Salbutamol (SAL) the bond length for C-C in a ring equal to $1.38 \AA$ and a side chin equal to $1.511 \AA$. The Propranolol (PRO) values are $1.37 \AA$ and 1.519 $\AA$ for the ring and the side chain. In the aromatic

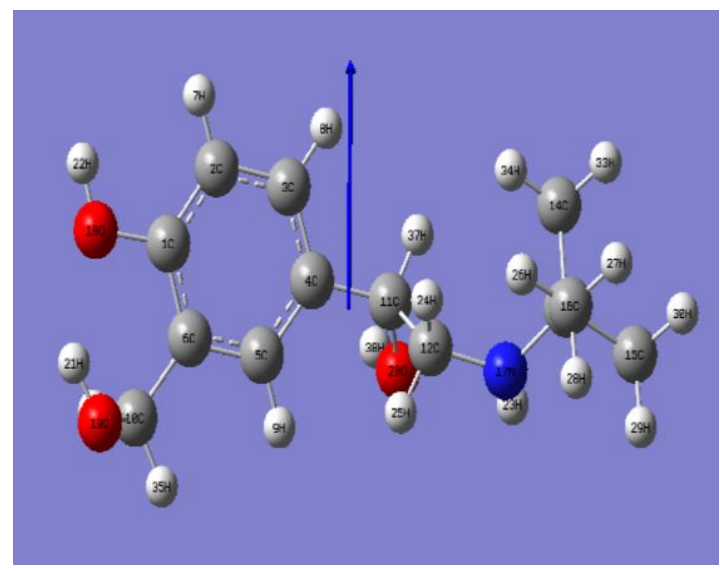

(A)

Tigure 2.8

\subsection{Frontier Molecular Orbitals}

Frontier Molecular Orbitals (FMOs) were used to estimate the most reactive position in the $\pi$ conjugated system and to describe many forms of reactions [26]. The energy values of the lowest unoccupied molecular orbital (LUMO) and the highest occupied molecular orbital (HOMO) and their energy difference $(\Delta \mathrm{E})$ represent the molecule's reactivity. HOMO and LUMO are stronger to determine the reactivity of the molecule, this also predicted the area of atomic electrophiles and nucleophiles, where [27]. (Figure 3) show HOMO and LUMO for both drugs were calculated using the B3LYP/6-31G $(\mathrm{d}, \mathrm{p})$ level. The (HOMO LUMO) energy bandgap represents the lowest electronic energy need to transfer an electron from $\pi-\pi *$. In the SAL compound, the maximum electronic energy (HOMO) displayed at $66^{\text {th }}$ is estimated at $-0.20258 \mathrm{eV}$, and the lowest ring (SAL) has only one ring, the $\mathrm{C}=\mathrm{C}$ bond length equal to $1.40 \AA$, But PRO has two rings, and the bond length equal to $1.42 \AA$. The big difference for bond length was appeared for C-N, for SAL C-N bond length equal to $2.57 \AA$, but PRO equal to $2.53 \AA$. Bond angles show strong cooperation between two drugs. For example, the bond angle between N17-C12-C11 is 112.7878 for SAL and is equal to 108.8592 for PRO. The dihedral angles indicate ring planarity. The dihedral for $\mathrm{C} 1, \mathrm{C} 2, \mathrm{C} 3$, C4 in SAL is -0.53458 , but for PRO is 1.15508 is means the PRO is more planer than SAL. The bond length and bond angle were demonstrating that PRO is more reactive than SAL.

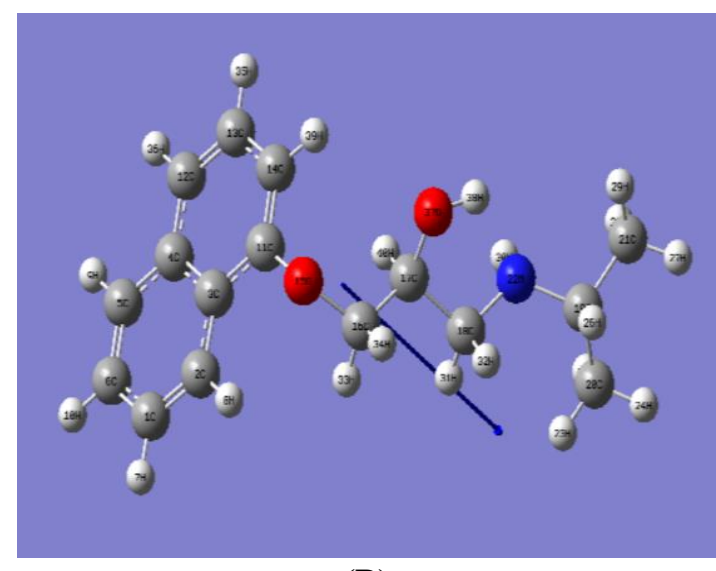

(B) 
equation $\omega=\mu^{2} / 2 \eta$ all data show in a Table 3 . and calculated using DFT at the basis set $6-31++\mathrm{G}$. The result of total energy, electrophilicity index and dipole moment of the PRO was shown the reactivity higher than the SAL compounds.

\subsection{Mulliken Charge Distribution}

Mulliken theory was used to calculate atomic charges and is defined in Table 4. The calculation was cared out on the DFT methods and $6-31++\mathrm{G}$ basis set, it is a lower theoretical energy level. In all SAL and PRO structures, the negative charge was distributed on atom specific in carbon, nitrogen, and oxygen. According to the result, SAL has a lower charge of nitrogen atoms. While most carbon

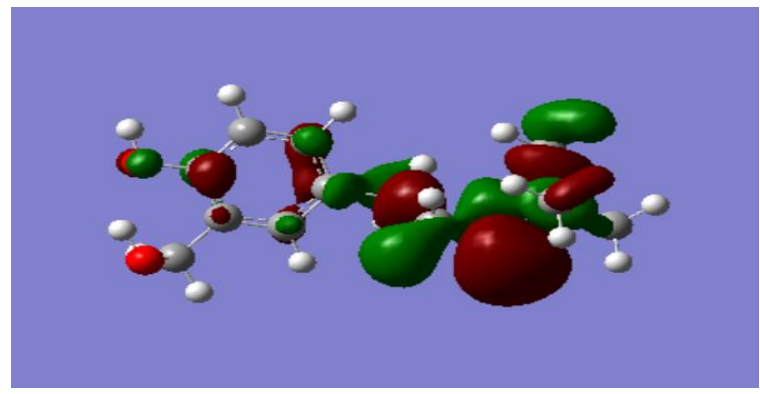

A) HOMO

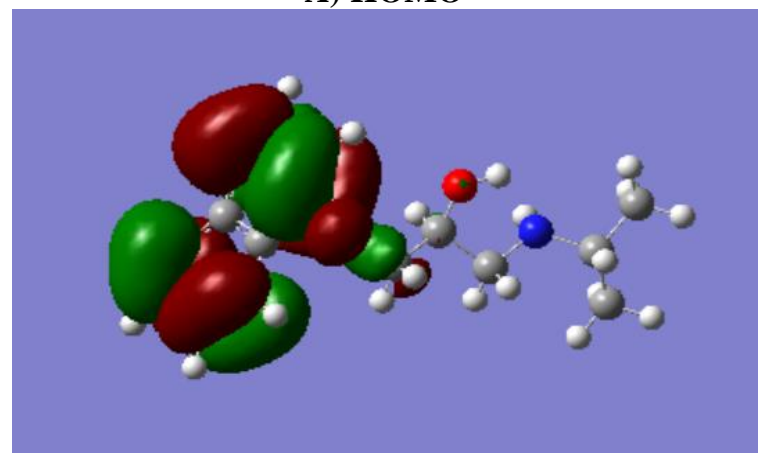

B) HOMO atom in SAL has a higher negative value compared with PRO. For oxygen atoms, SAL has three oxygen atom and the value of the negative charge was higher, while for PRO only two oxygen atoms in a structure and the negativity value was lower compared by SAL. Those values of the atomic charge distribution on the oxygen atoms imply that the portion of the structure has potential sites for interaction with poor electronic molecules. Although the charge distribution on the nitrogen atoms indicated that the structure lovely interacts with electrophilic species such as radicals. For the SAL structures more reactive with nucleophilic species, while PRO more reactive with electrophilic molecules.

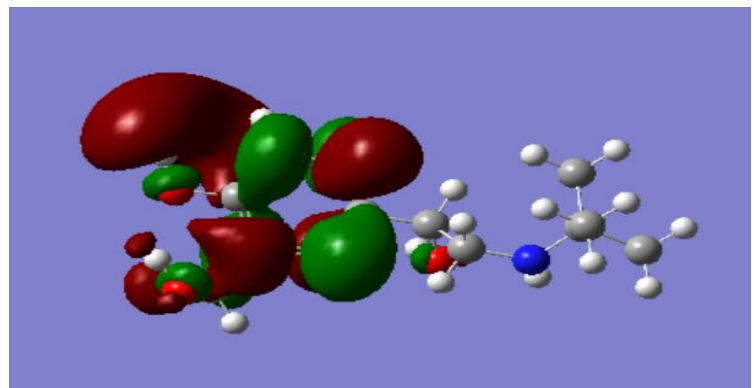

A) LUMO

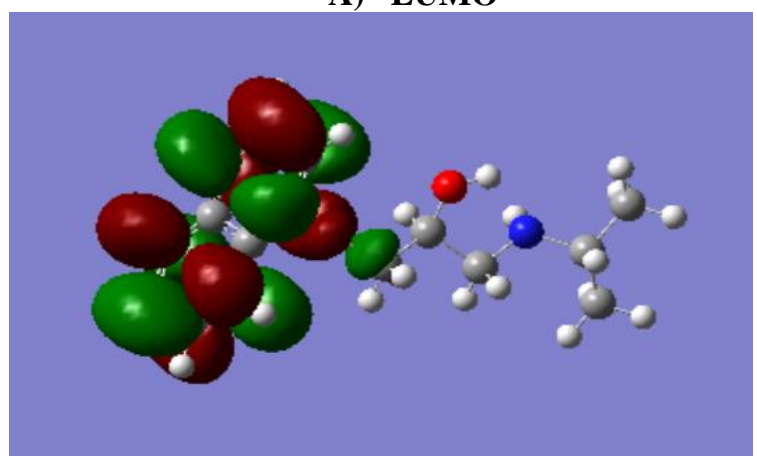

B) LUMO

Figure 3. Molecular orbital frontier surfaces for HOMO and LUMO (A) Salbutamol (SAL) (B) Propranolol (PRO), computed by B3LYP/6-31++G(d,p) level.

\subsection{Molecular Electrostatic Potential}

Charge spread on the surface of the molecules can be described and achieved electrostatic potential maps of surfaces. This map diagram enables us to visualize variable particle charged zones on a molecular surface. The advantages of the electrostatic potential map are to show how chemical interaction and chemical bonds between atoms were formed in a molecule. By using the molecular surface charging distribution, we now how molecules interact with other molecules. The molecule can be defined by an electrostatic diagram according to the scale of color. The red color suggested larger electrical density, and the distribution of electrons in this range is very condensed. However, the blue color range shows the reduced electronic density and the electronegativity is not very high. The distribution of charges is a difference in electronegativity and can determine the polarization of the molecule. The large electronegativity is distributed in red color then down to blue color. The MEP of the title compound was also determined by B3LYP/6$31++\mathrm{G}(\mathrm{d}, \mathrm{p})$ optimized geometry find the reactive 
Rebaz A. Omar,, Pelin Koparir, Lana O. Ahmed, Matin Koparir

site of the electrophilic and nucleophilicity. MEP's negative region red color more appeared in PRO molecule near to oxygen atom was linked to electrophilic reactivity responsible for intramolecular hydrogen bonding. Moreover, the positive area blue color seems in the ASL molecule corresponding to the reactivity of nucleophilic and responsibility to intermolecular hydrogen bonding. The most structure of PRO was green color without blue color this is proved that PRO structure is very reactive with nucleophilicity pieces (Figure 4).

Table 2. Comparative between SAL and PRO in some geometrical parameters

\begin{tabular}{|c|c|c|c|c|c|c|c|c|c|c|c|c|c|}
\hline & & & Salb & utamol & AL) & & & & & Pro & ranolol (PI & $\mathrm{RO})$ & \\
\hline Sy. \& & & & & Bond & Bond & & & & & & Bond & & \\
\hline NO. & NA & NB & $\mathrm{NC}$ & Length & Angle & Dihedral & NO. & NA & NB & $\mathrm{NC}$ & Length & & Dihedral \\
\hline $\mathrm{C} 1$ & & & & 1.38560 & & & $\mathrm{C} 1$ & & & & 1.37356 & & \\
\hline $\mathrm{C} 2$ & 1 & & & 1.39507 & & & $\mathrm{C} 2$ & 1 & & & 747 & & \\
\hline C3 & 2 & 1 & & 1.40106 & 119.2849 & & C3 & 2 & 1 & & 1.42426 & 120.6169 & \\
\hline $\mathrm{C} 4$ & 3 & 2 & 1 & 1.40304 & 120.7106 & -0.53458 & $\mathrm{C} 4$ & 3 & 2 & 1 & 1.439392 & 119.1873 & 1.15 \\
\hline C5 & 4 & 3 & 2 & 1.40 & & & C5 & 4 & 3 & 2 & 1.42602 & 118.5725 & \\
\hline C6 & 5 & 4 & 3 & 1.39928 & 21.8762 & 0.11 & C6 & 5 & 4 & 3 & 1.382093 & 120.9999 & 478 \\
\hline H7 & 2 & 1 & 6 & & & & H7 & 1 & 2 & 3 & & 24 & \\
\hline H8 & 3 & 2 & 1 & 1.08 & 119.3685 & 179. & $\mathrm{H} 8$ & 2 & 1 & 6 & 1.084243 & 120. & 91 \\
\hline H9 & 5 & 4 & 3 & 1.08601 & 119.0087 & 179.301 & H9 & 5 & 4 & 3 & 1.08 & 118. & 388 \\
\hline $\mathrm{C} 10$ & 6 & 5 & 4 & 1.5 & & & H10 & 6 & 5 & 4 & & & \\
\hline C11 & 4 & 3 & 2 & 1.51311 & 120.603 & -177 & C11 & 3 & 2 & 1 & 1.42 & 122 & -179.976 \\
\hline $\mathrm{C} 12$ & 11 & 4 & 3 & 1.5 & & & $\mathrm{C} 12$ & 4 & 3 & 2 & & & \\
\hline C13 & 12 & 11 & 4 & $2.5^{\circ}$ & 115.1 & & $\mathrm{C} 13$ & 12 & 4 & 3 & 17 & 120 & \\
\hline C14 & 13 & 12 & 11 & 1.55 & 95.7352 & & C14 & 11 & 3 & 2 & 45 & 121 & \\
\hline $\mathrm{C} 15$ & 13 & 12 & 11 & 1.5 & 134.6 & & & & 3 & 2 & & & \\
\hline C16 & 13 & 12 & 11 & 1.5462 & 96.60 & & $\mathrm{C} 16$ & 15 & 11 & 3 & 1.472316 & 118.205 & 712 \\
\hline N17 & 12 & 11 & 4 & 1.4 & & & & & & 11 & & & \\
\hline O18 & 1 & 2 & 3 & & & & & 17 & 16 & 15 & & & \\
\hline O19 & 10 & 6 & 5 & 1.4 & & & C19 & & 17 & 16 & 2.5 & 13 & 906 \\
\hline $\mathrm{O} 20$ & 11 & 4 & 3 & & & & $\mathrm{C} 20$ & & & 7 & & & \\
\hline $\mathrm{H} 21$ & 19 & 10 & 6 & 0.9 & & & $\mathrm{C} 21$ & 19 & 18 & 17 & 93 & 139 & 465 \\
\hline $\mathrm{H} 22$ & 18 & 1 & 2 & 0.9 & & & $\mathrm{~N} 22$ & & 17 & 16 & & & \\
\hline $\mathrm{H} 23$ & 17 & 12 & 11 & 1.01 & & & $\mathrm{H} 23$ & 20 & 19 & 18 & 02 & & 741 \\
\hline $\mathrm{H} 24$ & 12 & 11 & 4 & 1.0 & 108.5 & & $\mathrm{H} 24$ & 20 & 19 & 18 & 87 & 110 & 063 \\
\hline $\mathrm{H} 25$ & 12 & 11 & 4 & 1.09 & 106.9061 & & $\mathrm{H} 25$ & 20 & 19 & 8 & 506 & & \\
\hline H26 & 16 & 13 & 12 & & & & H26 & 19 & 18 & 17 & & 86 & 75 \\
\hline $\mathrm{H} 27$ & 16 & 13 & 12 & 1.09 & & & H27 & 21 & 19 & 18 & 1.05 & 11 & \\
\hline $\mathrm{H} 28$ & 16 & 13 & 12 & 1.09476 & 109.8778 & 82.7 & $\mathrm{H} 28$ & 21 & 19 & 18 & 1.098849 & 110 & .0484 \\
\hline $\mathrm{H} 29$ & 15 & 13 & 12 & 1.09 & 110.228 & & H29 & 21 & 19 & 18 & 1.0 & 514 & 927 \\
\hline H30 & 15 & 13 & 12 & 1.09 & & & H30 & 22 & 18 & 17 & 1.02 & & \\
\hline H31 & 15 & 13 & 12 & 1.09804 & & & H31 & 18 & 17 & 16 & 1.10 & 109.9287 & 436 \\
\hline H32 & 14 & 13 & 12 & 1.0968 & 110.5539 & & H32 & 18 & 17 & 16 & 731 & 485 & 269 \\
\hline H33 & 14 & 13 & 12 & 1.09752 & 110.756 & 158.6443 & H33 & 16 & 15 & 11 & 1.100056 & 109.0842 & -64.8244 \\
\hline H34 & 14 & 13 & 12 & 1.09698 & 111.1835 & & H34 & 16 & 15 & 11 & 1.09 & 103 & .225 \\
\hline H35 & 10 & 6 & 5 & 1.0906 & 110.371 & & H35 & 13 & 12 & 4 & & 120.1828 & -179.242 \\
\hline H36 & 10 & 6 & 5 & 1.09929 & 110.3372 & & H36 & 12 & 4 & 3 & 1.086591 & 118.8337 & -179.825 \\
\hline H37 & 11 & 4 & 3 & 1.0972 & 109.6081 & & $\mathrm{O} 37$ & 17 & 16 & 15 & 1.454903 & 109.0789 & 62.9552 \\
\hline H38 & 20 & 11 & 4 & 0.97798 & 110.9497 & 59.22907 & H38 & 37 & 17 & 16 & 0.98812 & 105.8473 & 156.592 \\
\hline & & & & & & & H39 & 14 & 11 & 3 & 1.084563 & 118.7805 & 178.832 \\
\hline & & & & & & & $\mathrm{H} 40$ & 17 & 16 & 15 & 1.101282 & 109.6224 & -56.1974 \\
\hline
\end{tabular}


Turkish Comp Theo Chem (TC\&TC), 4(2), (2020), 67-75

Rebaz A. Omar,, Pelin Koparir, Lana O. Ahmed, Matin Koparir

Table 3. Calculated energies, dipole moments (D), frontier orbital energies, and description of chemical reactivity of the compound.

\begin{tabular}{ccc}
\hline In a Basis Set B3LYP/6- & Salbutamol $($ SAL) & Propranolol (PRO) \\
31++G(d,p) & & -827.351 \\
$\boldsymbol{E}_{\text {Total }}$ & -788.251 & $-0.21565 \mathrm{eV}$ \\
$\boldsymbol{E}_{\text {HOMo }}$ & $-0.20258 \mathrm{eV}$ & $-0.04543 \mathrm{eV}$ \\
$\boldsymbol{E}_{\text {LUMO }}$ & $-0.03149 \mathrm{eV}$ & $-0.17022 \mathrm{eV}$ \\
Energy bandgaps & $-0.17109 \mathrm{eV}$ & $0.08511 \mathrm{eV}$ \\
Chemical hardness $(\eta)$ & $0.08554 \mathrm{eV}$ & 0.26108 \\
Electronegativity $(\chi)$ & 0.23407 & $-0.13054 \mathrm{~J} / \mathrm{mol}$ \\
Chemical potential $(\mu)$ & $-0.117035 \mathrm{~J} / \mathrm{mol}$ & 0.10010 \\
Electro-philicity index & 0.08006 & $5.1816 \mathrm{D}$ \\
Dipole moment & $5.5988 \mathrm{D}$ & \\
\hline
\end{tabular}

Table 4. Mulliken atomic charges distribution for Carbone, nitrogen, and oxygen atom for both Salbutamol SAL and Propranolol PRO

\begin{tabular}{cccc}
\hline & Salbutamol (SAL) & \multicolumn{2}{c}{ Propranolol (PRO) } \\
\hline Atom & Charge & Atom & Charge \\
C1 & -0.290482 & $\mathrm{C} 1$ & -0.1475 \\
C2 & 0.356001 & $\mathrm{C} 2$ & -0.41047 \\
C3 & -1.348922 & $\mathrm{C} 3$ & 0.789894 \\
C4 & -0.113902 & $\mathrm{C} 4$ & 0.26489 \\
C5 & 0.450631 & $\mathrm{C} 5$ & -0.29628 \\
C6 & 0.644403 & $\mathrm{C} 6$ & -0.26438 \\
C10 & -0.60156 & $\mathrm{C} 11$ & -0.5702 \\
C11 & -0.049199 & $\mathrm{C} 13$ & -0.28161 \\
C12 & -0.763036 & $\mathrm{C} 14$ & -0.32726 \\
C13 & -0.735001 & $\mathrm{O} 15$ & -0.04409 \\
C14 & -0.458386 & $\mathrm{C} 16$ & -0.24878 \\
C15 & -0.548753 & $\mathrm{C} 17$ & -0.51256 \\
C16 & -0.455336 & $\mathrm{C} 18$ & 0.084604 \\
N17 & -0.136877 & $\mathrm{C} 19$ & -0.81393 \\
O18 & -0.676989 & $\mathrm{C} 20$ & -0.31278 \\
O19 & -0.514333 & $\mathrm{C} 21$ & -0.53488 \\
O20 & -0.431806 & $\mathrm{~N} 22$ & -0.56634 \\
& & $\mathrm{O} 37$ & -0.35995 \\
& & & -0.47402 \\
\hline
\end{tabular}

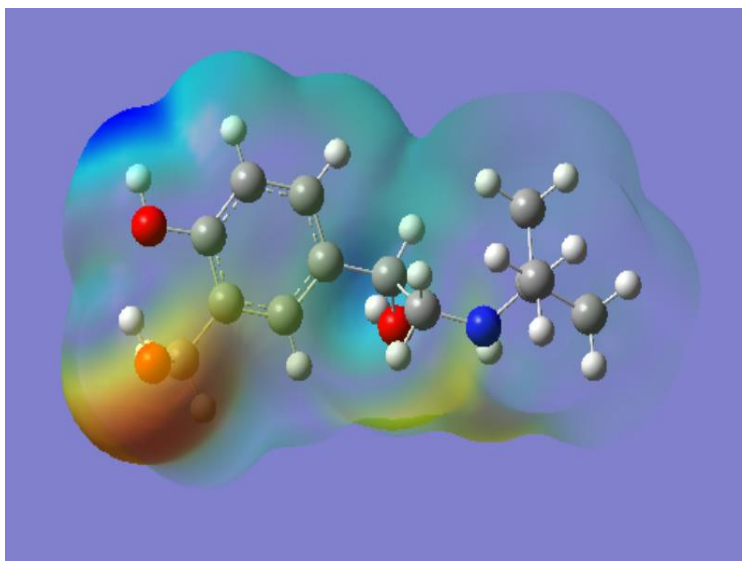

A

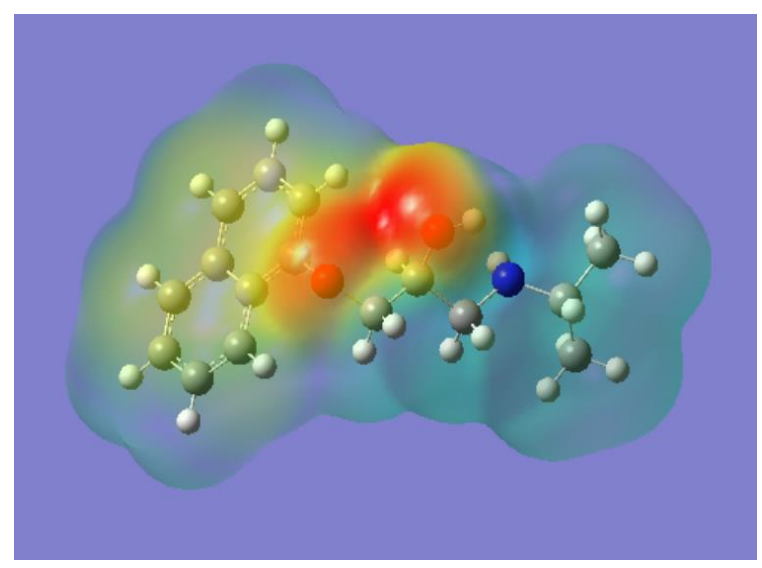

B

Figure 4. Electrostatic Potential Map of A) Salbutamol SAL and B) Propranolol PRO on a basis set B3LYP/6-31++G (d, p) 


\subsection{Thermodynamic Studies}

Table 5 revealed thermodynamic parameters for both SAL and PRO at semi-empirical parameters $\mathrm{AM} 1$ and $\mathrm{Ab}$ initio using two separate bases set $(\mathrm{HF} / 6-31++\mathrm{G}$ and B3LYP/6-31++G). It is clear that the AM1 base set was easily measured and faster compared to two other approaches with the saved computing times. The findings clearly demonstrate different total energy estimates and different energy levels for both drugs. Here, we took bout both semiempirical and $\mathrm{Ab}$ initio level parameters. The stability of the molecule is determined by the energy of the molecule, which included total energy, nuclear repulsion, electronic energy, and zero-point energy. Potential energy means molecular interaction and kinetic energy means molecular are formed in Table 5. In the present study, Using $A b$ initio on the basis set HF/6-31++G and B3LYP/6-31++G, the shift in all energies has been observed, increasing in value but with the same trend, implies that $\mathrm{PRO}$ is less reactive than SAL. Moreover, in the quantum mechanical system, the zero-point energy is the lowest possible energy are requiring. PRO displays a higher degree of zero-point energy in all basis sets and a better reactivity value than SAL. The estimation enthalpy and Gibbs free energy for SAL and PRO drugs are listed in Table 6. In a substance enthalpy is higher, means higher energy level. The lower energy level it is more reactive interacting with other substance. The enthalpy of PRO was higher in our sample according to all parameters and basis sets, it is more reactive than SAL. The Gibbs free energy was also higher for PRO than SAL, which is interpreted for SAL stability.

Table 5. Energies computed for Salbutamol SAL and Propranolol PRO (Kcal/mol).

\begin{tabular}{cccc}
\hline \multirow{2}{*}{ Energy (kcal/mol) } & Basis set & Salbutamol SAL & $\begin{array}{c}\text { Propranolol } \\
\text { PRO }\end{array}$ \\
\cline { 3 - 4 } & & & \\
\cline { 3 - 4 } Ab initio & & 229.821 & 239.955 \\
Thermal energy & HF/6-31++G & 217.933 & 227.970 \\
Nuclear repulsion & HFY/6-31++G & 768437.7875 & 863553.4465 \\
energy & B3LYP/6-31++G & 768437.8459 & 863553.4258 \\
Electronic energy & HF/6-31++G & -782.875638 & -821.602561 \\
& B3LYP/6-31++G & -787.922650 & -827.006821 \\
ZPE & HF/6-31++G & 220.26241 & 230.27916 \\
& B3LYP/6-31++G & 206.26817 & 216.53345 \\
\hline
\end{tabular}

Table 6. Calculate enthalpy and entropy Salbutamol SAL and Propranolol PRO (Kcal/mol)

\begin{tabular}{cccc}
\hline \multirow{2}{*}{ Parameters } & \multirow{2}{*}{$(\mathrm{Kcal} / \mathrm{mol})$ Base } & \multicolumn{2}{c}{ Structure } \\
\cline { 3 - 4 } Enthalpy & $\mathrm{HF} / 6-31++\mathrm{G}$ & 230.41337 & 240.547023 \\
& $\mathrm{~B} 3 \mathrm{LYP} / 6-31++\mathrm{G}$ & 218.525201 & 228.562217 \\
& & & \\
Gibbs Free Energy & $\mathrm{HF} / 6-31++\mathrm{G}$ & 194.306468 & 203.644438 \\
& $\mathrm{~B} 3 \mathrm{LYP} / 6-31++\mathrm{G}$ & 176.813382 & 186.096132 \\
\hline
\end{tabular}




\section{Conclusion}

To obtain energy bandgaps for SAL and PRO using both HF and DFT methods at different basis sets. B3LYP/6-31++G was choosing for all studies to determine the reactivity for both drugs (SAL \&PRO). PRO is reactive geometry with higher bond length compared with SAL. Calculated total energies, dipole moments, and frontier orbital energies were denoted the PRO structure have higher reactive than SAL due to less bandgap energy. The atomic charges distribution and molecular electrostatic potential (MEP) was determined to look at the higher electron density areas as possible interaction sites, such as nitrogen and oxygen. The PRO structure is very reactive with nucleophilicity pieces, but the SAL structure is reactive with electrophilicity according to charge distribution and (MEP). The tests of thermodynamics showed that the PRO is less stable than SAL. The collectivity data showed SAL to be more stable than PRO.

\section{References}

[1] L.K. Lundblad, et al., Detrimental effects of albuterol on airway responsiveness requires airway inflammation and is independent of $\beta$-receptor affinity in murine models of asthma. Respiratory research, 12 (2011) 27.

[2] S.E. Libretto, A review of the toxicology of salbutamol (albuterol). Archives of toxicology, 68 (1994) 213-216.

[3] S. Keir, C. Page, and D. Spina, Bronchial hyperresponsiveness induced by chronic treatment with albuterol: Role of sensory nerves. Journal of allergy and clinical immunology, 110 (2002) 388-394.

[4] D. Jarvie, A. Thompson, and E. Dyson, Laboratory and clinical features of selfpoisoning with salbutamol and terbutaline. Clinica chimica acta, 168 (1987) 313-322.

[5] S.M. Barkiya, et al., Effects of Aerosolized Levosalbutamol Verses Salbutamol on Serum Potassium Level and Heart Rate in Children with Acute Exacerbation of Asthma. INTERNATIONAL JOURNAL OF SCIENTIFIC STUDY, 3 (2016) 223227.
[6] D. Shand, Pharmacokinetics of propranolol: a review. Postgraduate medical journal, 52 (1976) 22-25.

[7] N. Tuross and R.L. Patrick, Effects of propranolol on catecholamine synthesis and uptake in the central nervous system of the rat. J Pharmacol Exp Ther, 237 (1986) 73945.

[8] A. Scriabine, B. Clineschmidt, and C. Sweet, Central noradrenergic control of blood pressure. Annual review of pharmacology and toxicology, 16 (1976) 113-123.

[9] J. Fallowfield and H. Marlow, Propranolol is contraindicated in asthma. BMJ: British Medical Journal, 313 (1996) 1486.

[10] D.J. Spitz, An unusual death in an asthmatic patient. The American journal of forensic medicine and pathology, 24 (2003) 271272.

[11] K. Albouaini, et al., Beta-blockers use in patients with chronic obstructive pulmonary disease and concomitant cardiovascular conditions. International journal of chronic obstructive pulmonary disease, 2 (2007) 535.

[12] N. Minton, A. Baird, and J. Henry, Modulation of the effects of salbutamol by propranolol and atenolol. European journal of clinical pharmacology, 36 (1989) 449453.

[13] E.A. Ramoska, et al., Propranolol treatment of albuterol poisoning in two asthmatic patients. Annals of emergency medicine, 22 (1993) 1474-1476.

[14] S. Küpeli, Use of propranolol for infantile hemangiomas. Pediatric hematology and oncology, 29 (2012) 293-298.

[15] A.M. Santos, A. Wong, and O. FatibelloFilho, Simultaneous determination of salbutamol and propranolol in biological fluid samples using an electrochemical sensor based on functionalized-graphene, ionic liquid and silver nanoparticles. Journal of Electroanalytical Chemistry, 824 (2018) 1-8.

[16] L. Liu, et al., Simultaneous determination of a broad range of cardiovascular drugs in plasma with a simple and efficient extraction/clean up procedure and 
chromatography-mass spectrometry analysis. RSC Advances, 4 (2014) 1962919639.

[17] S. Zhou, et al., Simultaneous separation of eight $\beta$-adrenergic drugs using titanium dioxide nanoparticles as additive in capillary electrophoresis. Electrophoresis, 29 (2008) 2321-2329.

[18] H.J. Azeez and V.S. Abdullah, Synthesis and Characterization of a New Series of Arylidene Compounds from 2Iminothiazolidine-4-one derivatives. ZANCO Journal of Pure and Applied Sciences, 31 (2019) 97-108.

[19] R. Ghavami and A. Navaee, Determination of nimesulide in human serum using a glassy carbon electrode modified with $\mathrm{SiC}$ nanoparticles. Microchimica Acta, 176 (2012) 493-499.

[20] A.M. Santos, et al., Square-wave voltammetric determination of paracetamol and codeine in pharmaceutical and human body fluid samples using a cathodically pretreated boron-doped diamond electrode. Journal of the Brazilian Chemical Society, 26 (2015) 2159-2168.

[21] H. Parham and B. Zargar, Determination of isosorbide dinitrate in arterial plasma, synthetic serum and pharmaceutical formulations by linear sweep voltammetry on a gold electrode. Talanta, 55 (2001) 255262.

[22] H.O. Ahmad, Computational study of optical properties, and enantioselective synthesis of di-substituted esters of hydantoic and thiohydantoic acids. Zanco Journal of Pure and Applied Sciences, 32 (2020) 75-94.

[23] L.A. Omer and O. Rebaz, Computational Study on Paracetamol Drug. Journal of Physical Chemistry and Functional Materials, 3 (2020) 9-13.

[24] L. Ahmed, R. Omer, and H. Kebiroglu, A theoretical study on Dopamine molecule. Journal of Physical Chemistry and Functional Materials, 2 (2019) 66-72.

[25] K. Katin, Benchmark Study of the Exchange-Corrected Density Functionals: Application to Strained Boron Nitride Clusters. Turkish Computational and Theoretical Chemistry, 1 27-34.

[26] D. Contreras, et al., Synthesis and Structural Determination of a New Chalcone 1, 5-Bis (3-Methyl-2-Thienyl) penta-1, 4-dien-3one, C15H14OS2. Journal of the Chilean Chemical Society, 54 (2009) 470-472.

[27] L. Padmaja, et al., Density functional study on the structural conformations and intramolecular charge transfer from the vibrational spectra of the anticancer drug combretastatin-A2. Journal of Raman Spectroscopy: An International Journal for Original Work in all Aspects of Raman Spectroscopy, Including Higher Order Processes, and also Brillouin and Rayleigh Scattering, 40 (2009) 419-428. 